\title{
A CONSTRUCTION FOR FITTING FORMATIONS II
}

\author{
JOHN COSSEY
}

(Received 21 December 1987)

Communicated by H. Lausch

\begin{abstract}
A construction for Fitting formations given by the author and C. L. Kanes is generalised. The original examples were based on the use of Fitting families of modules over algebraically closed fields. An example of Haberl and Heineken in 1984 suggested that the methods should work with modules over arbitrary fields. We show that this is indeed the case, provided we restrict the class of groups considered.
\end{abstract}

1980 Mathematics subject classification (Amer. Math. Soc.) (1985 Revision): 20 F 17.

\section{Introduction}

In [1] a general construction for Fitting formations and examples using that construction were given. The examples given were based on the use of Fitting families of modules, and the particular examples of Fitting families constructed relied on the use of modules over an algebraically closed field. It seemed clear these results could be extended to give examples using modules over non-algebraically closed fields, and the appearance of the example of Haberl and Heineken [2] provided the incentive to do so, as it seemed to be a $G F(q)$ version of the examples of [1]. However it turns out that the example of Haberl and Heineken can be constructed as a special case of the examples of [1] (see Section 4). The purpose of this paper is to show that the results of [1] can be extended to deal with modules over non-algebraically closed fields if we restrict the class of groups considered.

(C) 1989 Australian Mathematical Society 0263-6115/89 \$A2.00+0.00 


\section{Preliminaries}

For the remainder of this paper, $K$ will denote a perfect field of characteristic $q, q$ a fixed prime, and $E$ will denote the algebraic closure of $K$. We will denote by $\mathscr{P}$ a partition of $\mathbf{P}$, the set of all primes: so that $\mathscr{P}=\left\{\pi_{i} \mid i \in I\right\}$ for some index set $I$, with $\bigcup_{i \in I} \pi_{i}=\mathbf{P}$, and $\pi_{i} \cap \pi_{j}=\varnothing$ for $i \neq j$.

If $n$ is a positive integer prime to $q$ and $C_{n}$ a cyclic group or order $n$, we let $d_{K}(n)$ be the dimension of a faithful irreducible module for $C_{n}$ over $K$. We then set

$$
\left(K, \pi_{i}\right)=\left\{p \in \pi_{i} \mid p=q, d_{K}(p)=1, \text { or } d_{K}(p) \text { is not a } \pi_{i}^{\prime} \text {-number }\right\},
$$

and $(K, \mathscr{P})=\bigcup_{i \in I}\left(K, \pi_{i}\right)$. Note that if $K$ is algebraically closed, then $\left(K, \pi_{i}\right)$ $=\pi_{i}$, and so $(K, \mathscr{P})=P$. In general this will not be true, but for any field $K$ (except $G F(2)$ ) there is a nontrivial partition $\mathscr{P}$ of $\mathbf{P}$ with $(K, \mathscr{P})=\mathbf{P}$. Such a partition can be constructed by using the method given for $G F(q)$ by Haberl and Heineken in [2]. We indicate how their method can be adapted, and start with a restatement of their Hypothesis $B$.

HYPOTHEsIs $B$. $\mathfrak{R}_{K}$ is a set of positive integers satisfying

(a) $a b \in \mathfrak{R}_{K}$ if and only if $a$ and $b$ are both elements of $\mathfrak{R}_{K}$; and

(b) if $p$ is a prime, then $p \in \mathfrak{R}_{K}$ if and only if $d(p) \in \mathfrak{R}_{K}$.

Now define $\mu_{0}=\left\{p \in \Pi \mid p \neq q, d_{K}(p)=1\right\}$, and then $\mathfrak{M}_{0}$ to be the set of all $\mu_{0}$-numbers (note that $\mu_{0}$ is non-empty unless $K=G F(2)$ ). Then suppose that $\mu_{i}, \mathfrak{M}_{i}$ have been defined, and define $\mu_{i+1}, \mathfrak{M}_{i+1}$ as follows. If $\mathfrak{M}_{i}$ satisfies Hypothesis $\mathrm{B}$, set $\mu_{i+1}=\mu_{i}, \mathfrak{M}_{i+1}=\mathfrak{M}_{i}$, Otherwise, choose $m_{i}$ in $\mathfrak{M}_{i}$ minimal such that there is a prime $p$ not in $\mu_{i}$ with $d_{K}(p)=m_{i}$; and then let $\mu_{i+1}=\mu_{i} \cup\left\{p \in \Pi \mid d_{K}(p)=m_{i}\right\}$, and let $\mathfrak{M}_{i+1}$ be the set of all $\mu_{i+1^{-}}$ numbers. Finally, set $\mathfrak{M}_{K}=\bigcup \mathfrak{M}_{i}, \mu_{K}=\bigcup \mu_{i}$, and note that $q \notin \mu_{K}$. It is now an easy check that $\mathfrak{M}_{K}$ satisfies Hypothesis B (as in [2]).

Put $\mathscr{P}=\left\{\mu_{K}, \mu_{K}^{\prime}\right\}$. Then $\mathscr{P}$ is a nontrivial partition of $\mathbf{P}$, and we get as immediate consequences of the fact that $\mathfrak{M}_{K}$ satisfies Hypothesis $B$ that $\left(K, \mu_{K}\right)=\mu_{K}$ and $\left(K, \mu_{K}^{\prime}\right)=\mu_{K}^{\prime}$.

We will be interested in the structure of $K G$-modules for soluble $(K, \mathscr{P})$ groups $G$ : most of our results will be derived from analogous results for algebraically closed fields by extending $K$ to a finite extension which is a splitting field for $G$ and all its subgroups. We will need some facts about such extensions which we collect here.

Let $n$ be a $(K, \mathscr{P})$-number, coprime to $q$, and let $L=K(\varepsilon) \subset E$, where $\varepsilon$ is a primitive $n$th root of unity. Let $A=\mathrm{Gal}(L: K)$, the Galois group of $L$ over $K$ : then $A$ is abelian. If $\pi \in \mathscr{P}$, we can write $A=A_{1} \times A_{2}$, where $A_{1}$ is a $\pi$-group, and $A_{2}$ is a $\pi^{\prime}$-group. If $L_{i}$ is the fixed field of $A_{i}, i=1,2$, then $\operatorname{dim}_{L_{i}}(L)=\left|A_{i}\right|$, and $\operatorname{dim}_{K}\left(L_{i}\right)=\left|A_{j}\right|, j \neq i$. 
We will need the following results.

2.1 LeMMA. If $p \in(K, \pi)$, then $p \in\left(L_{1}, \pi\right)$.

Proof. If $p=q, q \in \pi$, and the result is immediate from the definition. If $K$ contains a $p$ th root of unity, so does $L_{1}$, and so we assume that $p \neq q$ and $d_{K}(p)>1$. Thus if $M$ is a faithful irreducible module for $C_{p}$ over $K, \operatorname{dim}_{K} M$ is not a $\pi^{\prime}$-number. If $M^{L_{1}}=M_{1} \oplus \cdots \oplus M_{t}$, with the $M_{i}$ irreducible, then the $M_{i}$ form an orbit under the action of $\operatorname{Gal}\left(L_{1}: K\right)$. Since $\left|\operatorname{Gal}\left(L_{1}: K\right)\right|=\left|A_{2}\right|$ is a $\pi^{\prime}$-number, so is $t$. Since $\operatorname{dim}_{K} M$ is not a $\pi^{\prime}$-number, it follows that $\operatorname{dim}_{L_{1}} M_{1}$ is not a $\pi^{\prime}$-number, and so $p \in\left(L_{1}, \pi\right)$.

2.2 Lemma. Suppose that $\alpha \in L_{1}$ is an nth root of unity, with $n a(K, \pi)$ number and coprime to $q$. Then $\alpha \in K$.

Proof. Clearly it is enough to prove the lemma for $n$ a prime power, $n=$ $p^{r}$ say, $p \in(K, \pi)$. In this case, any irreducible $K\langle\alpha\rangle$-module has dimension 1 or dimension divisible by $d_{K}(p)$.

Put $M=k(\alpha)$ : then $M$ is an irreducible $K\langle\alpha\rangle$-module. Since $\operatorname{dim}_{K} M$ divides $\operatorname{dim}_{K} L_{1}$, a $\pi^{\prime}$-number, $\operatorname{dim}_{K} M$ is not divisible by $d_{K}(p)$, and so $\operatorname{dim}_{K} M=1$. Thus $\alpha \in K$, as required.

\section{Factorable modules}

In [1], an irreducible $K G$-module $U$ was called $\mathscr{P}$-factorable if $U \cong$ $U_{1} \otimes \cdots \otimes U_{n}$, where $U_{j}$ is $\pi_{i_{j}}$-special, $\pi_{i_{j}} \in \mathscr{P}, \pi_{i_{j}} \neq \pi_{i_{k}}$ for $i_{j} \neq i_{k}, 1 \leq j$, $k \leq n$. The definition of $\pi$-special given in [1] does not require $K$ to be algebraically closed, but most of the results proved there do require the algebraic closure of $K$ : most of the results can be proved for an arbitrary perfect field $K$ provided we restrict ourselves to soluble $(K, \mathscr{P})$-groups. For the applications of $\mathscr{P}$-factorable modules needed for this paper it turns out to be more efficient to go in a different direction. Our aim in this section is to establish that, for soluble $(K, \mathscr{P})$-groups, a $K G$-module $M$ is $\pi$-special $(\mathscr{P}$-factorable) if and only if the irreducible constituents of $M^{E}$ are $\pi$-special ( $\mathscr{P}$-factorable). The same techniques can be used to establish analogues of the results of Section 2 of [1].

For the remainder of this section, if $G$ is a soluble group, $G_{q^{\prime}}$ will denote a Hall $q^{\prime}$-subgroup of $G, n=\left|G_{q^{\prime}}\right|, \varepsilon$ will denote a primitive $n$th root of unity in $E$, and $L=K(\varepsilon)$ (note that $L$ is a splitting field for every subgroup of $G$ ). Further, we set $A=\operatorname{Gal}(L: K)$, and if $\pi \in \mathscr{P}$, we put $A=A_{1} \times A_{2}$, where $A_{1}$ 
is a $\pi$-group and $A_{2}$ is a $\pi^{\prime}$-group. We let $F$ be the fixed field of $A_{2}$, so that $\operatorname{dim}_{K} F=\left|A_{1}\right|$ is a $\pi$-number.

3.1 Theorem. Let $G$ be a soluble $(K, \mathscr{P})$-group and $U \in \operatorname{Irr}_{K}(G)$. Then $U$ is $\pi$-special if and only if the irreducible constituents of $U^{E}$ are $\pi$-special.

Proof. Suppose first that $U$ is not $\pi$-special, but that the irreducible constituents of $U^{E}$ are $\pi$-special; and that $G$ has been chosen minimal with an irreducible $K G$-module with this property. Then by the minimality of $G$, if $S \triangleleft \triangleleft G$, the irreducible constituents of $U_{S}$ are $\pi$-special for $S \neq G$. Moreover for $s \in G$ with $s$ a $\pi^{\prime}$ element, $\operatorname{det}(s$ on $U)=\operatorname{det}\left(s\right.$ on $\left.U^{E}\right)=1$, since $U^{E}$ is a direct sum of $\pi$-special modules. Thus we must have that $\operatorname{dim}_{K} U$ is not a $\pi$-number.

Let $N$ be a maximal normal subgroup of $G$ : then $U_{N}$ is a direct sum of $\pi$ special modules (and so in particular $U_{N}$ cannot be irreducible). If $U_{N}$ is not homogeneous, we must have $U_{N}=V_{1} \oplus \cdots \oplus V_{t}$, where the $V_{i}$ are irreducible, and $t$ is a prime and the $\pi^{\prime}$ part of $\operatorname{dim}_{K} U$. Moreover $U \cong V_{1}^{G}$. Now suppose $V_{1}^{E}=W_{1} \oplus \cdots \oplus W_{l}$ : then $U^{E}=\left(W_{1} \oplus \cdots \oplus W_{l}\right)^{G}=W_{1}^{G} \oplus \cdots \oplus W_{l}^{G}$. Since $V_{1}$ is not $G$-invariant, $W_{i}$ is not $G$-invariant and so $W_{i}^{G}$ is irreducible, $i=1, \ldots, l$. But then the irreducible constituents of $U^{E}$ are not $\pi$-special since $\operatorname{dim}_{E} W_{i}^{G}$ is divisible by $t$ and is not a $\pi$-number.

Thus we suppose that $U_{N}$ is homogeneous. We let $\operatorname{dim}_{K} U=s t$, where $s$ is a $\pi$-number, and $t$ is a $\pi^{\prime}$-number. Then $U^{E}=U_{1} \oplus \cdots \oplus U_{l}$ (so that $t$ divides $l$ ) and $U_{N}=V_{1} \oplus \cdots \oplus V_{m}$, with the $V_{i}$ irreducible, $\pi$-special, and all isomorphic (so that $t$ divides $m$ ). Now suppose that $V_{1}^{E}=V_{1,1} \oplus \cdots \oplus V_{1 r}$, where the $V_{1 j}$ are irreducible and distinct, and that $\left(U_{1}\right)_{N}=U_{i 1} \oplus \cdots \oplus U_{i u}$, where the $U_{i j}$ are irreducible and all isomorphic. We observe that $U_{N}^{E}$ is isomorphic to the direct sum of $m$ copies of $V_{1}^{E}$, and that $U_{N}^{E}$ is isomorphic to the direct sum of $u$ copies of $U_{1,1} \oplus U_{2,1} \oplus \cdots \oplus U_{l 1}$. It follows that $r=l$, $u=m$, and so $l m$ divides $\operatorname{dim}_{K} U$. But $t$ divides both $l$ and $m$ and so $t^{2}$ divides $\operatorname{dim}_{K} U$, a contradiction.

Thus $U$ is $\pi$-special if the irreducible constituents of $U^{E}$ are $\pi$-special.

In the other direction, we begin by showing that the irreducible constituents of $U^{F}$ are $\pi$-special if $U$ is $\pi$-special. Thus suppose that $V$ is an irreducible constituent of $U^{F}$. Since $U^{F}$ is a direct sum of Galois conjugates of $V$, $\operatorname{dim}_{F} V$ is a $\pi$-number. For $S \triangleleft \triangleleft G$, let $W$ be an irreducible constituent of $V_{S}$. We then have that $W$ is isomorphic to an irreducible constituent of $X^{F}$, for some irreducible constituent $X$ of $U_{S}$. Suppose that $X^{F}=X_{1} \oplus \cdots \oplus X_{l}$, with $X_{1} \cong W$. Since $\operatorname{dim}_{K} X$ is a $\pi$-number, so are $\operatorname{dim}_{F} X_{i}$ and $l$. If $s$ is a $\pi^{\prime}$-element of $S$, we have $\left(X_{1}\right)_{\langle s\rangle}=X_{1,1} \oplus \cdots \oplus X_{1 m}$, where $X_{1 j}$ is irreducible, $j=1, \ldots, m$. We suppose the $X_{1 j}$ have been chosen so that $X_{1,1}, \ldots, X_{1 n}$ 
have dimension one, and $X_{1, n+1}, \cdots, X_{1 m}$ have dimension greater than one. For $j>n$, we have $\operatorname{det}\left(s\right.$ on $\left.X_{1 j}\right)=1$. For $j \leq n, s$ acts on $X_{1 j}$ as a root of unity $\alpha_{j} \in F$ of order a $\left(K, \pi^{\prime}\right)$-number. It then follows from Lemma 2.2 that $\alpha_{j} \in K$. Thus, letting $\sigma$ run over a tranversal for the stabilizer of $X_{1}$ in $\operatorname{Gal}(F: K)$, we have

$$
\begin{aligned}
1 & =\operatorname{det}(s \text { on } X)=\prod \operatorname{det}\left(s \text { on } X_{i}\right)=\prod \operatorname{det}\left(s \text { on } X_{1} \sigma\right) \\
& =\prod\left(\prod \alpha_{j}\right) \sigma=\left(\prod \alpha_{j}\right)^{l} .
\end{aligned}
$$

Since $l$ is a $\pi$-number and hence coprime to the order of $s$, we get $\operatorname{det}\left(s\right.$ on $\left.X_{1}\right)=\prod \alpha_{j}=1$. Thus we have that $W \cong X_{1}$ is $\pi$-special.

To complete the proof, it will be enough to show that $W$ is absolutely irreducible for $H$ a subgroup of $G$, and $W \in \operatorname{Irr}_{F}(H)$ with $\operatorname{dim}_{F} W$ a $\pi$ number. For then, if $V$ is an irreducible constituent of $U^{F}, V^{E}$ is irreducible, as is the extension to $E$ of each irreducible constituent of $V_{S}, S \triangleleft \triangleleft G$. It is then an easy check to see that $V^{E}$ is $\pi$-special. To see that $W$ is absolutely irreducible, it is enough to show that $W^{L}$ is irreducible. Suppose that $W^{L}=$ $W_{1} \oplus \cdots \oplus W_{k}$ : the $W_{i}$ form an orbit under the action of $A_{2}$. We then have that $k$ is a $\pi$-number (it is a divisor of $\operatorname{dim}_{F} W$ ) and a $\pi^{\prime}$-number (it is a divisor of $\left.\left|A_{2}\right|\right)$. Thus $k=1$, and the proof is complete.

3.2 THEOREM. Let $G$ be a soluble $(K, \mathscr{P})$-group, and $\pi \in \mathscr{P}$. If $U, U^{\prime}, V, V^{\prime}$ $\in \operatorname{Irr}_{K}(G)$ with $U, U^{\prime} \pi$-special and $V, V^{\prime} \pi^{\prime}$-special, then

(i) $U \otimes V$ is irreducible and

(ii) $U \otimes V \cong U^{\prime} \otimes V^{\prime}$ if and only if $U \cong U^{\prime}$ and $V \cong V^{\prime}$.

Proof. Let $U^{L}=U_{1} \oplus \cdots \oplus U_{t}$, with $U_{i}$ irreducible, $i=1, \ldots, t$. Since $t$ is a $\pi$-number, $A_{2}$ is contained in the stabilizer of each $U_{i}$ and $U_{i}=U_{1} \tau_{i}$ for some $\tau_{i} \in A_{1}$. Similarly, if $V^{L}=V_{1} \oplus \cdots \oplus V_{r}$, with the $V_{i}$ irreducible, $r$ is a $\pi^{\prime}$-number. Thus each $V_{i}$ is stabilized by $A_{1}$ and $v_{i}=V_{1} \sigma_{i}$ for some $\sigma_{i} \in A_{2}$. We then have

$$
(U \otimes V)^{L}=\bigoplus_{i, j}\left(U_{i} \otimes V_{j}\right)=\bigoplus_{i, j}\left(U_{1} \tau_{i} \otimes V_{1} \sigma_{j}\right)=\bigoplus_{i, j}\left(U_{1} \otimes V_{1}\right) \tau_{i} \sigma_{j}
$$

Since $U_{1}, V_{1}$ are absolutely irreducible $U_{1}^{E}, V_{1}^{E}$ are $\pi$-special and $\pi^{\prime}$-special respectively by Theorem 3.1. The irreducibility of $U_{1} \otimes V_{1}$ follows immediately from [1, Theorem 2.4]. Thus $(U \otimes V)^{L}$ is the sum of distinct Galois conjugates of an irreducible $L G$-module, and so $U \otimes V$ is irreducible.

If $U \otimes V \cong U^{\prime} \otimes V^{\prime}$, then $(U \otimes V)^{E} \cong\left(U^{\prime} \otimes V^{\prime}\right)^{E}$ and so for irreducible constituents $W, X, W^{\prime}, X^{\prime}$ of $U^{E}, V^{E}, U^{\prime}, V^{\prime E}$ respectively, we have 
$W \otimes X \cong W^{\prime} \otimes X^{\prime}$. From [1, Theorem 2.4] again we deduce that $W \cong W^{\prime}$, $X \cong X^{\prime}$, and hence $U \cong U^{\prime}, V \cong V^{\prime}$, as required.

An easy induction gives us the following corollary.

3.3 CoRollary. Let $U_{i}, V_{i}$ be $\pi_{j_{i}}$ special modules, $\pi_{j_{i}} \in \mathscr{P}, \pi_{j_{i}} \neq \pi_{j_{k}}$ for $i \neq k, 1 \leq i, k \leq n$. Then $U_{1} \otimes \cdots \otimes U_{n}$ is irreducible, and if $U_{1} \otimes \cdots \otimes U_{n} \cong$ $V_{1} \otimes \cdots \otimes V_{n}$, then $U_{i} \cong V_{i}, 1 \leq i \leq n$.

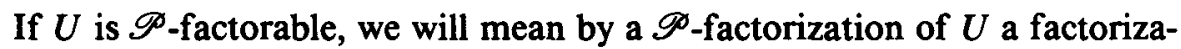
tion $U=u_{1} \otimes \cdots \otimes U_{n}$, with $U_{i}$ being $\pi_{j_{i}}$-special and not the trivial irreducible, $\pi_{j_{i}} \in \mathscr{P}, \pi_{j_{i}} \neq \pi_{j_{k}}$, for $i \neq k, 1 \leq i, k \leq n$.

3.4 TheORem. Let $G$ be a soluble $(K, \mathscr{P})$-group, and $U \in \operatorname{Irr}_{K}(G)$. Then $U$ is $\mathscr{P}$-factorable if and only if the irreducible constituents of $U^{E}$ are $\mathscr{P}$ factorable.

Proof. Suppose first that $U$ is $\mathscr{P}$-factorable, with $U=U_{1} \otimes \cdots \otimes U_{n}$ a $\mathscr{P}$-factorization of $U$. If $U_{i}^{E}=U_{i, 1} \oplus \cdots \oplus U_{\mathrm{im}_{i}}$ we get that $U^{E}$ is a direct sum of modules of the form $U_{1 j_{1}} \otimes \cdots \otimes U_{n j_{n}}$. It follows from Theorem 3.1 and Corollary 3.3 that the $U_{1 j_{1}} \otimes \cdots \otimes U_{n j_{n}}$ are the irreducible constituents of $U^{E}$, and so the irreducible constituents of $U^{E}$ are $\mathscr{P}$-factorable.

Now suppose that $V$ is an irreducible constituent of $U^{E}$, with $V$ being $\mathscr{P}$-factorable and $V=V_{1} \otimes \cdots \otimes V_{n}$ a $\mathscr{P}$-factorization of $V$. Let $W_{i}$ be an irreducible $K G$-module such that $W_{i}^{E}$ has an irreducible constituent isomorphic to $V_{i}$. Since Galois conjugates of $\pi_{j_{i}}$-special modules are clearly $\pi_{j_{i}}$-special, we have by Theorem 3.1 that $W_{i}$ is $\pi_{j_{i}}$-special. If we set $W=W_{1} \otimes \cdots \otimes W_{n}$, $W$ is irreducible by Corollary 3.3 , and then $U \cong W$, since $U^{E}$ and $W^{E}$ have a common composition factor. Thus $U$ is $\mathscr{P}$-factorable, as required.

The results above rely on the fact that we have restricted our attention to soluble $(K, \mathscr{P})$-groups: they do not hold if $(K, \mathscr{P})$ is replaced by a larger set of primes.

\section{Fitting formations}

We retain the notation of Section 2. For each $i \in I$, we let $\mathfrak{X}_{i}$ be a Fitting formation, and set $\mathscr{S}=\left\{\mathfrak{X}_{i} \mid i \in I\right\}$.

As in [1] we define for each soluble $(K, \mathscr{P})$-group $G$ a class of modules as follows:

$M_{K}(G)=\left\{M \in \operatorname{Irr}_{K}(G) \mid M\right.$ is $\mathscr{P}$-factorable, with $\mathscr{P}$-factorization

$$
\left.M=M_{1} \otimes \cdots \otimes M_{n} \text {, and } G / C_{G}\left(M_{i}\right) \in \mathfrak{X}_{j_{i}}, 1 \leq i, k \leq n\right\} .
$$


We then define a class of groups by

$$
\mathfrak{M}(K, \mathscr{P}, \mathscr{Z})=\left\{G \in \mathfrak{S}_{(K, \mathscr{P})} \mid \Gamma_{K}(G) \subseteq M_{K}(G)\right\} .
$$

Note that $\mathfrak{M}(E, \mathscr{P}, \mathscr{X})=\mathfrak{M}(q, \mathscr{P}, \mathscr{Z})$ in the notation of [1]. This seems to be the natural way to generalize the examples of [1, Section 4]: there is an alternative characterization of these classes which is easier to work with.

4.1 ThEOREM. $\mathfrak{M}(K, \mathscr{P}, \mathscr{X})=\mathfrak{M}(E, \mathscr{P}, \mathscr{X}) \cap \mathfrak{S}_{(K, \mathscr{P})}$.

Proof. Since $\mathfrak{M}(K, \mathscr{P}, \mathscr{Z}) \subseteq \mathfrak{S}_{(K, \mathscr{P})}$, it will be enough to show that for $G \in \mathfrak{S}_{(K, \mathscr{P})}$ and $U \in \operatorname{Irr}_{K}(G), U \in M_{K}(G)$ if and only if all the irreducible constituents of $U^{E}$ are in $\mathfrak{M}_{E}(G)$.

By Theorem 3.4 $U$ has a $\mathscr{P}$-factorization $U=U_{1} \otimes \cdots \otimes U_{n}$ if and only if $W$ has a $\mathscr{P}$-factorization $W=W_{1} \otimes \cdots \otimes W_{n}$, where $W$ is an irreducible constituent of $U^{E}$ and $W_{i}$ is isomorphic to an irreducible constituent of $U_{i}^{E}$. Since all the irreducible constituents of $U_{i}^{E}$ are Galois conjugates we have $C_{G}\left(U_{i}\right)=C_{G}\left(W_{i}\right)$, and so $G / C_{G}\left(U_{i}\right) \in \mathfrak{X}_{j_{i}}$ if and only if $G / C_{G}\left(W_{i}\right) \in \mathfrak{X}_{j_{i}}$. This completes the proof.

\subsection{COROLlaRY. $\mathfrak{M}(K, \mathscr{P}, \mathscr{Z})$ is a Fitting formation.}

We are interested in knowing which of these Fitting formations are saturated. Theorem 4.2 of [1] and its proof can be modified to give a complete answer: we leave it to the reader to make the appropriate changes.

The main result of the paper of Haberl and Heineken [2] is the following. Let $\mathfrak{C}$ be a class of finite soluble groups satisfying the following conditions:

(i) all finite nilpotent groups are in $\mathfrak{C}$;

(ii) there is a set $\mathfrak{R}_{q}$ of natural numbers such that $\mathfrak{C}$ is the class of groups with the rank of every $q$-chief factor in $\mathfrak{R}_{q}$;

and

(iii) $\mathfrak{C}$ is a Fitting class.

Haberl and Heineken show that there is for each odd prime $q$ exactly one non-trivial set (denoted by $\mathscr{M}$ in [2]) for which such a class $\mathfrak{C}$ exists; and this class is just the class $\mathfrak{M}_{G F(q)}$ defined in Section 2.

4.3 TheOREM. Let $\mathfrak{C}$ be the class determined by $\mathfrak{M}_{G F(q)}, \mu=\mu_{G F(q)}$ the set of primes defined in Section $2, \mathscr{P}=\left\{\mu, \mu^{\prime}\right\}$, and $\mathscr{Z}=\{\mathfrak{S}, \mathfrak{E}\}$. Then $\mathfrak{C}=\mathfrak{M}(G F(q), \mathscr{P}, \mathscr{X})$.

Proof. We have observed in Section 2 that $(G F(q), \mathscr{P})=\mathbf{P}$, and so $\mathfrak{M}(G F(q), \mathscr{P}, \mathscr{Z})$ is just the class of all soluble groups in which every $q$ chief factor is $\mu$-special. Clearly then $\mathfrak{M}(G F(q), \mathscr{P}, \mathscr{Q}) \subseteq \mathfrak{C}$. On the other hand, 
suppose that $G \in \mathbb{C}$ and $U=H / K$ is a $q$ chief factor of $G$. Then the rank of $U$ is a $\mu$-number. Moreover if $S \triangleleft \triangleleft G, V$ is an irreducible constituent of $U_{S}$, and $s \in S$ is a $\mu^{\prime}$-element, we have $\operatorname{det}(s$ on $V) \in G F(q)$. Thus if $\operatorname{det}(s$ on $V)$ is an $n$th root of unity, $n$ is a $\mu$-number and a $\mu^{\prime}$-number, giving $\operatorname{det}(s$ on $V)=1$. Hence $U$ is $\mu$-special, and $G \in \mathfrak{M}(G F(q), \mathscr{P}, \mathscr{Z})$, completing the proof.

It is worth noting that this example of Haberl and Heineken provided the incentive for extending the results of [1], as it seemed that the example should be obtainable from a $G F(q)$ version of the results in [1]: however it turns out that it can be realised as a special case of the examples of [1], for by Theorem 4.1 $\mathfrak{C}=\mathfrak{M}(E, \mathscr{P}, \mathscr{X})$ (with $\mathscr{P}, \mathscr{X}$ as in Theorem 4.3).

\section{References}

[1] J. Cossey and C. L. Kanes, 'A construction for Fitting formations', J. Algebra 107 (1987), 117-133.

[2] K. L. Haberl and H. Heineken, 'Fitting classes defined by chief factor ranks', J. London Math. Soc. (2) 29 (1984), 34-40.

Department of Mathematics

Australian National University

P. O. Box 4

A.C.T. 2601

Australia 